\title{
Clinical management of patients with gastric neuroendocrine neoplasms associated with chronic atrophic gastritis: a retrospective, multicentre study
}

\author{
Davide Campana $^{1}$ - Davide Ravizza ${ }^{2}$ Piero Ferolla ${ }^{3}$ - Antongiulio Faggiano ${ }^{4} \cdot$ \\ Franco Grimaldi ${ }^{5}$ - Manuela Albertelli ${ }^{6,7}$ - Debora Berretti ${ }^{5}$ - Danilo Castellani ${ }^{8}$. \\ Giulia Cacciari $^{1} \cdot$ Nicola Fazio $^{2} \cdot$ Annamaria Colao $^{4} \cdot$ Diego Ferone $^{6,7} \cdot$ \\ Paola Tomassetti ${ }^{1}$
}

Received: 10 January 2015/Accepted: 19 March 2015/Published online: 27 March 2015

(C) Springer Science+Business Media New York 2015

\begin{abstract}
To provide data regarding clinical presentation, pathological features, management, and response to different treatments of patients with type I gastric neuroendocrine tumors in stages $0-2 \mathrm{~A}$. The study design consist of an Italian multicentre, retrospective analysis of patients with type I gastric neuroendocrine tumors managed with different therapeutic approaches: surgery, endoscopic surveillance, endoscopic resection, or somatostatin analog therapy. Among the 97 patients included, 3 underwent surgery, $45(46.4 \%)$ radical endoscopic resection of the neoplastic lesions, $13(13.4 \%)$ follow-up with upper endoscopy, and $36(37.1 \%)$ somatostatin analog therapy. At
\end{abstract}

Davide Campana

davide.campana@unibo.it

1 Department of Medical and Surgical Sciences, S.OrsolaMalpighi University Hospital, University of Bologna, Via Massarenti, 9, 40138 Bologna, Italy

2 Division of Endoscopy and Unit of Gastrointestinal and Neuroendocrine Tumours, European Institute of Oncology, Milan, Italy

3 Department of Medical Oncology, Multidisciplinary NET Center, Umbria Regional Cancer Network, Umbria, Italy

4 Division of Endocrinology, Department of Clinical Medicine and Surgery, "Federico II" University of Naples, Naples, Italy

5 Endocrinology and Metabolism Unit, University Hospital S. Maria della Misericordia, Udine, Italy

6 Endocrinology, Department of Internal Medicine and Medical Specialties (DiMI) and Center of Excellence for Biomedical Research (CEBR), University of Genova, Genoa, Italy

7 IRCCS-AOU San Martino-IST, Genoa, Italy

8 Department of Gastroenterology, Multidisciplinary NET Center, Umbria Regional Cancer Network, Umbria, Italy the end of the follow-up, all patients were alive and there was no evidence of metastatic disease. Somatostatin analog therapy resulted in a complete response in $76.0 \%$ of the patients and stable disease in $24.0 \%$. A prolonged period of therapy, the use of a full dose of somatostatin analogs and higher gastrin levels at diagnosis were related to a complete response to the therapy. The recurrence rate was $26.3 \%$ in patients treated with somatostatin analog therapy and $26.2 \%$ in patients treated with endoscopic resection, without a statistically significant difference in terms of disease-free survival. Regarding recurrence of the disease, no statistical difference was found according to type of therapy, number of neoplastic lesions, and 2010 WHO classification. The only risk factor for tumor recurrence was a short period of medical treatment. In conclusion, our study suggested that endoscopic surveillance, endoscopic resection and somatostatin analog therapy represent valid options in the management of patients with type I gastric neuroendocrine tumors in stages $0-2 \mathrm{~A}$.

Keywords Neuroendocrine tumors - Chronic atrophic gastritis · Gastric carcinoid - Somatostatin analogs · Endoscopic resection

\section{Introduction}

Gastric neuroendocrine neoplasms (gNENs-also called "carcinoids") are tumors derived from enterochromaffinlike cells (ECL-cells) localized in the gastric mucosa [1].

Three subtypes of ECL-cell tumors have been recognized [2]: type I lesions (70-80\%) are associated with chronic atrophic gastritis (CAG) and hypergastrinemia; type II lesions (5-8 \%) are associated with gastrinomas in Zollinger-Ellison syndrome (ZES) and multiple endocrine 
neoplasia type I (MEN 1), and type III (15-20\%) are sporadic lesions arising in otherwise normal gastric mucosa without hypergastrinemia [2].

Neoplastic changes in ECL-cells (types I and II gNENs) are always associated with an elevated concentration of circulating serum gastrin [2], which exerts a trophic effect on ECL-cells, leading to hyperplasia and, in some cases, to a true neuroendocrine neoplasm [3, 4]. The majority of type I gNENs occur in women and are rarely symptomatic [5]. They are non-functioning tumors, typically found during upper gastrointestinal (GI) endoscopy for dyspepsia or anemia [6]. Type I gNENs frequently present as multiple polyps, usually $<1 \mathrm{~cm}$ in diameter, localized in the gastric corpus-fundus. They are almost exclusively benign lesions with a low risk of deep invasion of the gastric wall [7]. These tumors have a good prognosis with the 5-year survival rate quoted at $96 \%$ which does not differ from an age-matched normal population $[5,8]$.

Therapeutic strategies for type I gNENs (ranging from endoscopic surveillance to surgery) are based on risk stratification according to tumor size, lesion number, stage, and grade [5, 9, 10]. Current European Neuroendocrine Tumor Society (ENETS) guidelines for the management of patients with type I gNENs suggest endoscopic management with lesion resection [11], while a surgical approach should be limited to cases of clearly demonstrated invasion beyond the submucosa and/or with metastases. The current literature points out the elevated variability which exists between treatments in cases of multiple, localized (mucosa or submucosa) type I gNENs: (1) careful endoscopic follow-up without any treatment [12-14], (2) somatostatin analog (SSA) therapy $[9,15,16]$ and (3) endoscopic resection $[10,17]$. Two papers have recently been published in which a retrospective analysis of patients with type I gNENs treated with different approaches was carried out. Thomas et al. compared endoscopic resection with surgical treatment [18], while Sato et al. compared endoscopic surveillance with endoscopic resection [19].

The aim of this retrospective study was to provide data regarding the clinical presentation, pathological features, management, and response to different treatment modalities in a large cohort of patients with type I gNENs.

\section{Patients and methods}

\section{Study design and patients}

The study design consisted of an Italian multicentre, retrospective analysis. The study included all consecutive patients with type I gNENs followed at the participating centers (i.e., Bologna, Milan- IEO, Perugia, Naples, Udine, Genova) from June 1998 to December 2013.
Demographic data, as well as clinical and pathological features, were collected in a dedicated computerized database including: (1) baseline situations with demographic data, past medical history, symptoms at diagnosis of the gNEN, evaluations of parietal cell antibodies (PCA), serum gastrin levels and plasma/serum levels of Chromogranin A $(\mathrm{CgA})$, findings at endoscopy and endoscopic ultrasound (EUS), and the pathological features of gNENs and CAG; (2) type of therapy (surgery, endoscopic resection, SSAs, endoscopic surveillance) and (3) follow-up data and status at last visit.

\section{Data analysis}

A dedicated computerized database was created, and demographic data, as well as pathological features, were retrospectively analyzed. The histological specimens were examined by an expert NEN-dedicated pathologist at each centre. When required, additional centralized revision of the tumor specimens was carried out. The degree of atrophy of the gastric mucosa was classified according to the updated Sydney system classification [20].

The tumors were classified according to the 2010 WHO classification [21] and the tumor node metastasis (TNM) classification/G grading system [22]. The Ki67 proliferation index was expressed as a percentage based on the number of Ki67-positive cells in 2000 tumor cells in the areas of the highest immunostaining using the MIB1 antibody (DBA, Milan, Italy).

The response to therapy was classified as: complete response (CR): the disappearance of all signs (endoscopic and histologic) of gNEN; progressive disease (PD): stage increase of gNEN and stable disease (SD): no change in terms of gNEN stage. Disease-free survival (DFS) was defined as the interval between the endoscopic and pathologic evidence of being disease free and the time of disease recurrence (DR). Disease-free survival was measured using the Kaplan-Meier method, and the results were compared using the log-rank test. Analysis of the predictive risk factors for DR was carried out by univariate analysis using the Cox proportional hazards method. Risk factors were expressed as hazard ratios (HRs) [95\% confidence interval (CI)]. All analyses carried out for risk factors are listed in tables. The distribution of the continuous variables was reported as median and interquartile ranges (IQRs, 25th to 75 th percentiles). Comparison between the subgroups was carried out using Pearson's Chi-square test (Fisher's exact test was used when necessary) or the Mann-Whitney $U$ test for continuous variables. The $p$ value was considered significant when $<0.05$. The statistical analysis was carried out using dedicated software (IBM-SPSS Statistics version 19.0). 


\section{Results}

\section{Study population}

Ninety-seven patients with gNENs associated with CAG were enrolled at the participating centers. The characteristics of all 97 patients are listed in Table 1 . There were 38 males and 59 females with a median age of 59.0 years (IQR 49.0-66.0 years). In 24 patients (24.7\%), the diagnosis of gNEN was incidental, in $48(49.5 \%)$ as a result of dyspepsia and in $25(25.8 \%)$ as a result of anemia. Seventy-three $(75.3 \%)$ patients had autoimmune disease with positive PCA and 19 (19.6\%) with negative PCA (not evaluated in 5 patients). All patients had serum gastrin levels above the normal value (median: $964.0 \mathrm{pg} / \mathrm{ml}$; IQR: $585.0-1702.0 \mathrm{pg} / \mathrm{ml}$ ). The median $\mathrm{CgA}$ level, evaluated in 54 patients using enzyme-linked immunosorbent assay (ELISA), was 77.5 U/l (IQR: 41.0-198.0 U/l). In 25 patients, CgA evaluation was carried out using radioimmunoassay (RIA) (median: $216.0 \mathrm{pg} / \mathrm{ml}$; IQR: $110.0-353.0 \mathrm{pg} / \mathrm{ml}$ ). Therefore, $\mathrm{CgA}$ was not evaluated in 18 patients.

At upper gastrointestinal endoscopy, 60 patients $(61.9 \%)$ were found to have fewer than 5 polyps in the body-fundus and 37 (38.1\%) to have more than 5 lesions; the median lesion diameter was $5 \mathrm{~mm}$ (IQR 3-10 mm). Atrophy of the gastric mucosa was mild in 18 patients $(18.6 \%)$, moderate in $32(33.0 \%)$, and severe in 36 $(37.1 \%)$ an CAG without grade of atrophy was described in 11 patients $(11.3 \%)$. No patient had Helicobacter Pylori infection. According to the 2010 WHO classification, 56 patients $(57.8 \%)$ had a NET G1, $33(34.0 \%)$ a NET G2 and, in 8 patients, histological examination was not possible due to the scarcity of the tissue samples. The median Ki67 index was $2.0 \%$ (IQR 1.0-3.0\%). At diagnosis, 84 patients $(86.6 \%)$ were in stages $0-1,8(8.2 \%)$ in stage $2 \mathrm{~A}$ (lesions limited to the submucosa but having a diameter between 1 and $2 \mathrm{~cm}$ ) and $3(3.1 \%)$ in stage 3B (median lesion diameter: $30 \mathrm{~mm}$, range $25-35 \mathrm{~mm}$; 1 NET G1 and 2 NET G2; 2 T2N1 and 1 T3N1). Therefore, the stage of the disease was not evaluated in 2 patients.

\section{Tumor treatment}

The tumor treatment of the 97 patients is listed in Table 1; $45(46.4 \%)$ patients underwent radical endoscopic resection of the neoplastic lesions, $13(13.4 \%)$ were followed up by endoscopy, $36(37.1 \%)$ received SSA therapy with or without partial endoscopic resection [20 (20.6\%) patients were treated with SSAs only and $16(16.5 \%)$ with both partial endoscopic resection and SSAs]. In 3/97 $(3.1 \%)$ patients with stage $3 \mathrm{~B}$, radical surgery was carried out. As reported in Table 1, endoscopic resection was preferred in patients having fewer than 5 lesions $(p<0.001)$; SSA therapy was preferred in patients with NET G1s $(p=0.013)$ who had lower Ki67 $(p=0.017)$ and higher gastrin levels $(p=0.005)$.

The median follow-up was 30.5 months (IQR: 12.0-64.2 months). Sixty-four patients were disease free at follow-up (31 after endoscopic resection, 4 after a simple follow-up, 26 after somatostatin analog therapy and 3 after radical surgery); 14 had stable disease ( 9 after a simple follow-up, 5 after SSA therapy); 16 had a recurrence of the disease (11 after complete endoscopic resection and 5 after SSA therapy) and 3 were lost to follow-up. At the end of the follow-up period, all patients were alive and none presented metastatic disease (lymph node or liver).

In order to undergo SSA therapy, 9/36 patients gave their informed written consent, and the therapy was approved by the local Ethics Committee [9]. The remaining 27 patients were treated according to "extension of indication for uses (of somatostatin analogs) consolidated on the basis of scientific evidence in the literature."

The flowchart of patients therapy is shown in Fig. 1.

\section{Endoscopic surveillance}

Thirteen ( 6 males and 7 females, median age: 62 years, IQR: 50-65 years) patients were followed up, without any additional treatment, for a median period of 82.0 months (IQR: 34.0-120.0 months). In 2 patients, there were fewer than 5 lesions while, in 11 patients, there were more than 5 neoplastic lesions. The median lesion diameter was $4.5 \mathrm{~mm}$ (IQR: 3.0-10.0). Three patients had a NET G1 while $8 / 13$ had a NET G2; the median Ki67 level was $3 \%$ (IQR: 2.0-5.0). During follow-up, there was complete disappearance of the neoplastic lesions in 4 patients $(30.8 \%)$; in the remaining 9 cases, there was stable disease in terms of stage of the disease ( 8 stage I and 1 stage IIA).

\section{Endoscopic resection}

Forty-five (14 males and 31 females, median age: 61 years, IQR: 49-71 years) patients were treated with endoscopic resection (polypectomy, endoscopic mucosal dissection, endoscopic submucosal dissection). In 41 patients, there were fewer than 5 lesions while, in 4 patients, there were more than 5 neoplastic lesions. The median lesion diameter was $5.0 \mathrm{~mm}$ (IQR: $3.0-8.0$ ). Twenty-eight patients had a NET G1 while 16 had a NET G2; the median Ki67 level was $1.9 \%$ (IQR: $1.0-3.4$ ); $41 / 45$ had stages $0-1$ of the disease and 4 had stage 2A. During follow-up, there was DR (median 13 months; IQR 10-31 months) in 11 patients (24.4\%); 31 were DF (median follow-up: 17 months; IQR 12-38 months) while 3 patients were lost to follow-up. 
Table 1 General features at diagnosis of the 97 type I gNENs patients enrolled, classified according to type of tumor treatment

\begin{tabular}{|c|c|c|c|c|c|}
\hline & \multicolumn{5}{|l|}{$n(\%)$} \\
\hline & Overall & ER & ES & SSA & $p$ \\
\hline All patients & $97^{\mathrm{a}}$ & $45(46.4)$ & $13(13.4)$ & $36(37.1)$ & \\
\hline Sex & & & & & 0.387 \\
\hline Male & $38(39.2)$ & $14(38.9)$ & $6(16.7)$ & $16(44.4)$ & \\
\hline Female & $59(60.8)$ & $31(53.4)$ & $7(12.1)$ & $20(34.5)$ & \\
\hline Diagnosis & & & & & 0.727 \\
\hline Occasional & $24(24.7)$ & $11(45.8)$ & $5(20.8)$ & $8(33.3)$ & \\
\hline Dyspepsia & $48(49.5)$ & $22(46.8)$ & $5(10.6)$ & $20(42.6)$ & \\
\hline Anemia & $25(25.8)$ & $12(52.2)$ & $3(13.0)$ & $8(34.8)$ & \\
\hline PCA & & & & & 0.197 \\
\hline Yes & $73(75.3)$ & $32(45.1)$ & $8(11.3)$ & $31(43.7)$ & \\
\hline No & 19 (19.6) & $10(55.6)$ & $4(22.2)$ & $4(22.2)$ & \\
\hline Not evaluable & $5(5.2)$ & & & & \\
\hline Gastric polyps & & & & & $<0.001$ \\
\hline$<5$ & $60(61.9)$ & $41(69.5)$ & $2(3.4)$ & $16(27.1)$ & \\
\hline$>5$ & $37(38.1)$ & $4(11.4)$ & $11(31.4)$ & $20(57.1)$ & \\
\hline Intestinal metaplasia & & & & & 0.209 \\
\hline Yes & $85(88)$ & 37 (44.6) & $12(14.5)$ & $34(41.0)$ & \\
\hline No & $12(12)$ & $8(72.7)$ & $1(9.1)$ & $2(18.2)$ & \\
\hline Grade of atrophy & & & & & 0.194 \\
\hline Mild & 18 (18.6) & $11(61.1)$ & $2(11.1)$ & $5(27.8)$ & \\
\hline Moderate & $32(33.0)$ & $11(36.7)$ & $2(6.7)$ & $17(56.7)$ & \\
\hline Severe & $36(37.1)$ & $16(45.7)$ & $9(25.7)$ & $10(28.6)$ & \\
\hline Not specified & $11(11.3)$ & & & & \\
\hline 2010 WHO classification & & & & & 0.013 \\
\hline NET G1 & $56(57.8)$ & $28(50.9)$ & $3(5.5)$ & $24(43.6)$ & \\
\hline NET G2 & $33(34.0)$ & 16 (51.6) & $8(25.8)$ & 7 (22.6) & \\
\hline Not evaluable & $8(8.2)$ & & & & \\
\hline Stage & & & & & 0.432 \\
\hline $0 / 1$ & $84(86.6)$ & $41(48.8)$ & $9(10.7)$ & $34(40.5)$ & \\
\hline $2 \mathrm{~A}$ & $8(8.2)$ & $4(50.0)$ & $2(25.0)$ & $2(25.0)$ & \\
\hline $3 \mathrm{~B}$ & $3(3.1)$ & 0 & 0 & 0 & \\
\hline Not evaluable & $2(2.1)$ & & & & \\
\hline & Median (IQR) & & & & \\
\hline Gastrin $(\mathrm{pg} / \mathrm{dl})$ & $964.0(585.0-1702.0)$ & $843.0(440.0-1511.0)$ & $829.0(387.5-950.5)$ & $1521.0(749.0-2540.0)$ & 0.005 \\
\hline $\mathrm{CgA}(\mathrm{U} / \mathrm{l})$ & $77.5(41.0-198.0)$ & $79.0(41.0-213.0)$ & $188.5(61.0-210.0)$ & $62.0(37.0-84.0)$ & 0.178 \\
\hline Ki67 \% & $2.0(1.0-3.0)$ & $1.9(1.0-3.4)$ & $3.0(2.0-5.0)$ & $1.2(1.0-2.4)$ & 0.017 \\
\hline Lesion diameter $(\mathrm{mm})$ & $5.0(3.0-10.0)$ & $5.0(3.0-8.0)$ & $4.5(3.0-10.0)$ & $5.0(3.0-8.0)$ & 0.668 \\
\hline
\end{tabular}

Bold values are statistically significant $(p<0.05)$

gNEN gastric neuroendocrine neoplasia, ER endoscopic resection, ES endoscopic surveillance, $S S A$ somatostatin analog therapy, $P C A$ parietal cell antibodies, $\mathrm{CgA}$ chromogranin A

a Three patients with stage $3 \mathrm{~B}$ underwent a surgical procedure (partial or total gastrectomy)

\section{Somatostatin analog therapy}

Thirty-six patients $(37.1 \%)$ were treated with long-acting SSAs. In 11/36 (30.5\%) patients, there was no evidence of residual disease after endoscopy (complete endoscopic resection) while, in 25/36 (69.5\%), there was evidence of residual disease (the presence of at least one neoplastic lesion histologically proven and not removed). The characteristics of these 25 patients are listed in Table 2. Nineteen patients (76\%) had a complete response after a median period of 
45 patients were treated with

radical endoscopic resection.

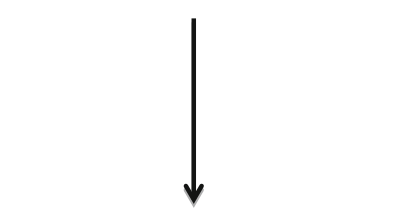

11 patients $\rightarrow$ disease relapsed

(median 13 months)

31 patients $\rightarrow$ disease free

(median 17 months)

3 patients $\rightarrow$ lost to follow-up
11 patients were treated after a complete endoscopic resection

36 patients were treated with

somatostatin analogues.

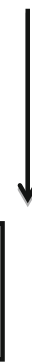

18 patients were treated with somatostatin analogues only.

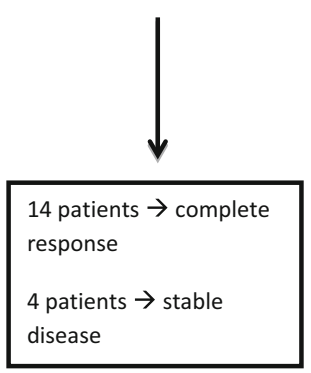

13 patients were followed up by endoscopy.

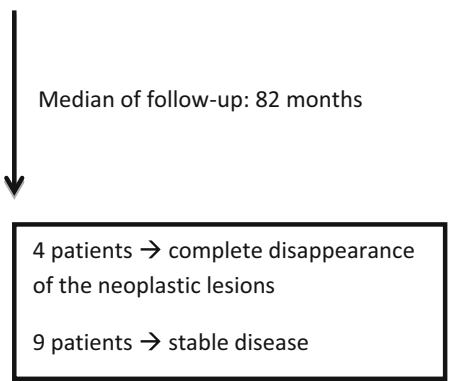

Fig. 1 Flowchart of patients therapy

therapy of 15 months (IQR: 12.0-24.0) and 6 (24\%) had stable disease after median period of therapy of 7 months (IQR: $5.0-11.0 ; p=0.024$ ). Eighteen out of nineteen $(94.7 \%)$ patients having a complete response were administered a full dose of SSAs (Lanreotide Autogel $120 \mathrm{mg}$ or Octreotide LAR $30 \mathrm{mg}$ every 28 days) and the remaining patient a lower dose of SSAs (Lanreotide Autogel $60 \mathrm{mg}$ every 28 days); $3 / 6$ (50\%) patients with stable disease were administered a full dose of SSA and 3 a lower dose of SSA (Octreotide LAR $20 \mathrm{mg}$ every 28 days; $p=0.031$ ). The gastrin serum levels at diagnosis were significantly higher in patients with a complete response (1882.0 vs. $954.0 \mathrm{pg} / \mathrm{ml}$; $p=0.039$ ). No differences were found between complete response and stable disease regarding sex, the presence of PCA, the number of gastric polyps, the grade of atrophy, 2010 WHO classification, the stage of the disease, the associated partial endoscopic resection, Ki67 \% and lesion diameter.

Of the 11 patients without evidence of residual disease after endoscopy who were treated with SSAs, 5/11 were being treated with SSAs at the moment of the study (median length of therapy: 36 months; IQR 31-60) without DR, 5/11 were not being treated with SSR therapy and had DR (median length of therapy: 14 months, IQR 13-21; median follow-up: 34 months; IQR 30-49) and one patient had DR after 69 months of therapy.

\section{Predictors of tumor recurrence and disease-free survival}

Of the 64 patients having a complete response after therapy, 3 were lost to follow-up; $42 / 61(68.8 \%)$ were treated with endoscopic resection while $19 / 61(31.1 \%)$ were treated with SSAs. Sixteen patients $(26.2 \%)$ had disease recurrence [11/42 $(26.2 \%)$ after endoscopic resection and $5 / 19(26.3 \%)$ after medical therapy]. The median DFS was 117 months (Fig. 2). No statistical difference was found according to $\operatorname{sex}(p=0.347)$, type of therapy (medical: median not reached, endoscopic resection: median 117 months; $p=0.644$ ), number of neoplastic lesions (less than 5: median 117 months, more than 5: median not reached; $p=0.448$ ), 2010 WHO classification (NET G1: median not reached, NET G2: median 117 months; $p=0.433)$ and grade of atrophy $(p=0.528)$.

The variables considered as risk factors for tumor recurrence after medical or endoscopic treatment are summarized in Table 3. The only risk factor for tumor recurrence was a short period of medical treatment (HR $0.38, p=0.024)$. In particular, no recurrence was found in patients when the period of medical treatment was 17 months or longer (Fig. 3-median follow-up = 62 months, IQR: 20.5-68.5). 
Table 2 General features of 25 type I gNENs treated with somatostatin analogs, classified according to tumor response

\begin{tabular}{|c|c|c|c|c|}
\hline & \multicolumn{4}{|l|}{$n(\%)$} \\
\hline & Overall & Complete response & Stable disease & $p$ \\
\hline All patients & 25 & $19(76.0)$ & $6(24.0)$ & \\
\hline Sex & & & & 0.661 \\
\hline Male & 11 & $9(81.8)$ & $2(18.2)$ & \\
\hline Female & 14 & $10(71.4)$ & $4(28.6)$ & \\
\hline PCA & & & & 1.000 \\
\hline Yes & 20 & $15(75.0)$ & $5(15.0)$ & \\
\hline No & 4 & $3(75.0)$ & $1(15.0)$ & \\
\hline Not evaluable & 1 & & & \\
\hline Gastric polyps & & & & 0.562 \\
\hline$<5$ & 5 & $3(60.0)$ & $2(40.0)$ & \\
\hline$>5$ & 20 & $16(80.0)$ & $4(20.0)$ & \\
\hline Grade of atrophy & & & & 0.519 \\
\hline Mild & 2 & $1(50.0)$ & $1(50.0)$ & \\
\hline Moderate & 11 & $10(90.9)$ & $1(9.1)$ & \\
\hline Severe & 8 & $5(62.5)$ & $3(37.5)$ & \\
\hline Not specified & 4 & & & \\
\hline 2010 WHO classification & & & & 0.407 \\
\hline NET G1 & 18 & $12(66.7)$ & $6(33.3)$ & \\
\hline NET G2 & 4 & $4(100.0)$ & 0 & \\
\hline Not evaluable & 3 & & & \\
\hline Stage & & & & 0.240 \\
\hline $0 / 1$ & 24 & $19(79.2)$ & $5(20.8)$ & \\
\hline $2 \mathrm{~A}$ & 1 & 0 & $1(100.0)$ & \\
\hline Associated polipectomy & & & & 1.000 \\
\hline Yes & 7 & $5(71.4)$ & $2(28.6)$ & \\
\hline No & 18 & $14(77.8)$ & $4(22.2)$ & \\
\hline Dosage of SSAs & & & & $\mathbf{0 . 0 3 1}$ \\
\hline Low-dose & 4 & $1(33.3)$ & $3(66.7)$ & \\
\hline Full dose & 21 & $18(83.3)$ & $3(16.7)$ & \\
\hline & Median (IQR) & & & \\
\hline Gastrin (pg/dl) & $1521.0(1042.0-2551.0)$ & $1882.0(1392.0-2631.0)$ & $954.0(749.0-1224.0)$ & $\mathbf{0 . 0 3 9}$ \\
\hline Ki67 \% & $1.0(0.8-2.4)$ & $1.3(0.9-2.5)$ & $1.0(0.8-2.0)$ & 0.316 \\
\hline Lesion diameter $(\mathrm{mm})$ & $5.0(3.0-9.0)$ & $5.0(3.0-8.0)$ & $5.0(3.0-10.0)$ & 0.846 \\
\hline Months of therapy & $13.0(10.5-22.0)$ & $15(12-24)$ & $7(5-11)$ & $\mathbf{0 . 0 2 4}$ \\
\hline
\end{tabular}

Bold values are statistically significant $(p<0.05)$

gNENs gastric neuroendocrine neoplasias, PCA parietal cell antibodies, SSAs somatostatin analogs

\section{Discussion}

Therapeutic strategies for type I gNENs, ranging from endoscopic surveillance to surgery, are based on risk stratification according to tumor size, number of neoplastic lesions, stage, and grade $[5,9,10]$. The current ENETS guidelines for the management of patients with type I gNENs suggest endoscopic management with lesion resection [11] while a surgical approach should be limited to cases of invasion beyond the submucosa and/or with nodal involvement.
In this multicentre retrospective study, the outcome of three main therapeutic approaches were analyzed in 94 patients with type I gNENs: endoscopic resection (46.4 \%), endoscopic surveillance (13.4\%), and SSA therapy (37.1\%).

The study showed that, in patients with stages $0-2 \mathrm{~A}$ type I gNENs, endoscopic surveillance, endoscopic resection, or SSA therapy were all feasible and potentially effective; no nodal or distant metastases occurred and there was $100 \%$ survival at the end of the follow-up. 


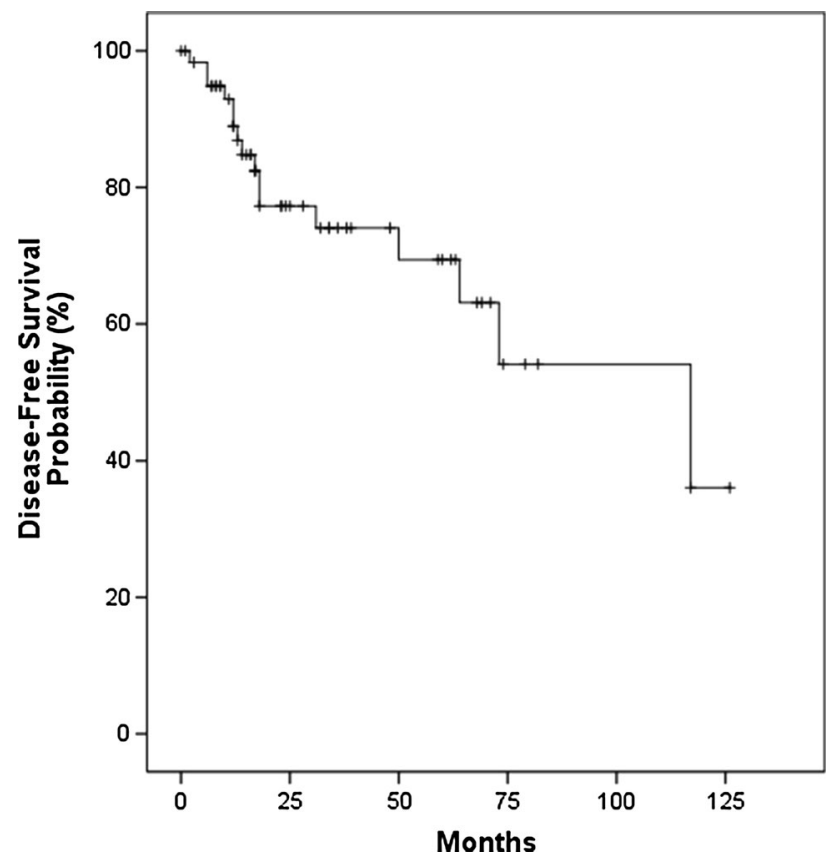

Fig. 2 Disease-free survival in 61 patients radically treated for type I gNENs

Table 3 The effects of various clinical, radiologic, and histologic findings on tumor recurrence in 61 patients with type I gNENs

\begin{tabular}{llll}
\hline & \multicolumn{3}{l}{ Univariate } \\
\cline { 2 - 4 } & HR & $95 \%$ CI & $p$ value \\
\hline Age at diagnosis & 0.99 & $0.96-1.04$ & 0.965 \\
Female gender & 1.71 & $0.55-5.31$ & 0.355 \\
Positive PCA & 1.66 & $0.37-7.55$ & 0.511 \\
More than 5 polyps & 0.66 & $0.23-1.94$ & 0.453 \\
Grade of Atrophy & & & \\
$\quad$ Mild & 1 & & \\
Moderate & 3.00 & $0.36-25.14$ & 0.310 \\
$\quad$ Severe & 2.02 & $0.23-17.67$ & 0.524 \\
NET G2 & 1.52 & $0.53-4.42$ & 0.438 \\
Treatment & & & \\
Endoscopic vs medical & 1.28 & $0.44-3.70$ & 0.646 \\
Gastrin & 1.00 & $1.00-1.00$ & 0.181 \\
Ki67 \% & 1.04 & $0.85-1.29$ & 0.693 \\
Lesions diameter & 0.94 & $0.79-1.12$ & 0.508 \\
Months of therapy & 0.38 & $0.19-0.88$ & $\mathbf{0 . 0 2 4}$ \\
\hline
\end{tabular}

Bold value is statistically significant $(p<0.05)$

gNENs gastric neuroendocrine neoplasias, $P C A$ parietal cell antibodies, $H R$ hazard ratio, $C I$ confidence interval

The use of SSA therapy in type I gNENs is still under debate. In the literature, there are prospective studies involving small numbers of patients which have reported the efficacy of SSA therapy in a specific setting of patients

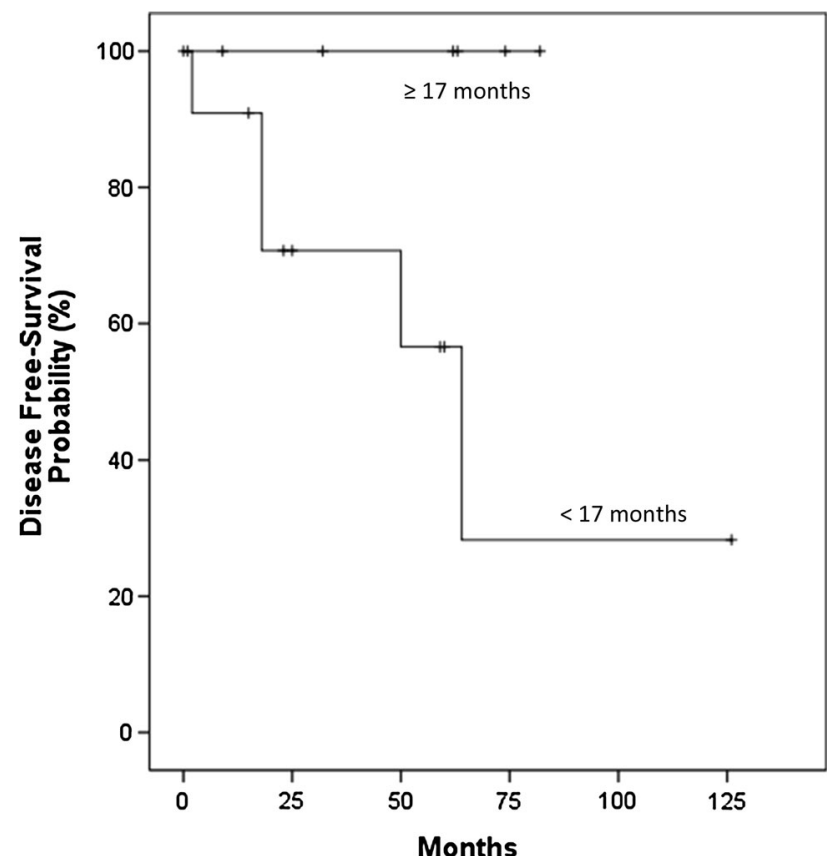

Fig. 3 Disease-free survival in 19 complete response patients treated with SSA, according to the period of the therapy

with type 1 gNENs [9, 15]. Given the absence of prospective randomized trials, according to the ENETS guidelines, the use of SSAs is still under debate and should be proposed only in accordance with expert opinion.

According to the literature, SSA therapy produced a CR of $76.0 \%$ and SD of $24.0 \%$ with no cases of progression $[9,15,23,24]$. These data underscore the potential role of these drugs in this setting due to their antiproliferative effect and the reduction of gastrin levels $[9,25]$. Our data suggest the importance of using a full dose of SSAs (Lanreotide Autogel $120 \mathrm{mg}$ or Octreotide LAR $30 \mathrm{mg}$ every 28 days) and a prolonged period of therapy in order to obtain a complete response. To our knowledge, no data regarding these aspects have previously been reported.

An additional finding provided by this study was a relatively low recurrence rate. In particular a disease recurrence of $26.3 \%$ occurred in patients treated with SSAs and $26.2 \%$ in patients treated with endoscopic resection without a statistically significant difference in terms of disease-free survival (median: 117 months). Another observation in our study not previously reported in the literature was that there was the same risk of disease recurrence using SSAs and endoscopic resection.

Regarding the recurrence rate after endoscopic resection, our data showed a lower percentage as compared with those of Merola et al. who reported a $63.6 \%$ recurrence rate after endoscopic resection using a forceps $(54.4 \%)$ or by snare $(45.5 \%)$ with a recurrence-free survival of 
24 months [10]. On the other hand, in a recent series of 22 patients with type I gNENs undergoing endoscopic mucosal resection (EMR "lift and cut"), Uygun et al. described an $18.2 \%$ recurrence rate with a median follow-up of 7 years [17]. These experiences suggest that the type of endoscopic procedure used to remove the neoplastic lesions (forceps, endoscopic mucosal resection or endoscopic submucosal dissection) could play a central role in defining the risk of recurrence. Prospective, comparative studies are needed to investigate this aspect.

Studying predictors of disease recurrence shows that only prolonged medical therapy has a positive prognostic role. In particular, medical treatment for 17 months or longer seemed to prevent the risk of recurrence with a median follow-up of up to 62 months. Our data are the first to suggest a minimal period for therapy in patients treated with SSAs.

A possible limitation of SSA therapy or endoscopic submucosal dissection is the high cost of these therapeutic options. Only a specific cost/effectiveness study can evaluate this aspect.

In our retrospective analysis, endoscopic surveillance, carried out in 13/94 patients, seemed to be a reasonable alternative to medical or endoscopic therapy. In fact, stable disease or disappearance of the disease (4 cases) was not observed after a median follow-up of 82.0 months. This evidence confirmed the data in the literature which indicated the potential role of endoscopic surveillance in selected patients with type I gNENs [14].

It is noteworthy that, in our study, all the patients treated with a conservative strategy (endoscopic surveillance, endoscopic resection or SSA therapy) had stage 0 or stage I lesions. Patients with stage $2 \mathrm{~A}$ lesions had tumor lesions limited to the submucosa having a diameter between 1 and $2 \mathrm{~cm}$. The 2 cases with T2 lesions, having a diameter greater than $2 \mathrm{~cm}$, had lymph node involvement at endoscopic ultrasound (stage 3B). Regarding this aspect, La Rosa et al. described the statistically significant increased risk of tumor metastases in patients with invasion of the deep submucosa, also with the presence of a lesion diameter between 1 and $3 \mathrm{~cm}$ [26]. As a result of these data, in our opinion, it is useful to carry out an endoscopic ultrasound evaluation in all patients with a lesion diameter $>1 \mathrm{~cm}$ or with a lesion diameter $<1 \mathrm{~cm}$ but with deep submucosal invasion (shown histologically) in order establish the neoplastic extension into the gastric wall and the perigastric lymph node status prior to establishing the therapeutic approach.

The possible limitations of our study include different time intervals of endoscopic follow-up, the lack of a centralized revision of the pathological specimens and a heterogeneous thefrapeutic approach (type of endoscopic resection, and type and duration of SSA therapy). Despite this aspect, our retrospective study reports some interesting data and presents many ideas for future prospective studies.

In conclusion, our study suggests that endoscopic surveillance, endoscopic resection, and SSA therapy are all valid options in the management of patients with type I gNENs in stages $0-2 \mathrm{~A}$. The risk of recurrence is the same in patients treated with endoscopic resection and SSA therapy. In patients treated with SSAs, at least 17 months of medical therapy is recommended in order to increase the possibility of obtaining a complete response and of reducing the risk of disease recurrence.

Although type I gNENs are quite rare, prospective randomized trials to compare the different therapeutic options and to evaluate the cost of these options are warranted.

Acknowledgments ALL authors approved the final version of the article, including the authorship list. Dr. Antongiulio Faggiano has received research grants from NOVARTIS Farma s.p.a. and IPSEN PHARMA. The others authors declare that they have no conflict of interest.

\section{References}

1. M.D. Burkitt, D.M. Pritchard, Review article: pathogenesis and management of gastric carcinoid tumours. Aliment. Pharmacol. Ther. 24, 1305-1320 (2006)

2. G. Rindi, O. Luinetti, M. Cornaggia, C. Capella, E. Solcia, Three subtypes of gastric argyrophil carcinoid and the gastric neuroendocrine carcinoma: a clinicopathologic study. Gastroenterology 104, 994-1006 (1993)

3. H.L. Waldum, A.K. Sandvik, J.R. Idle, Gastrin is the most important factor in ECL tumorigenesis. Gastroenterology 114, 1113-1115 (1998)

4. T. Lehy, A.M. Roucayrol, M. Mignon, Histomorphological characteristics of gastric mucosa in patients with Zollinger-Ellison syndrome or autoimmune gastric atrophy: role of gastrin and atrophying gastritis. Microsc. Res. Tech. 48, 327-338 (2000)

5. K. Borch, B. Ahren, H. Ahlman, S. Falkmer, G. Granerus, L. Grimelius, Gastric carcinoids: biologic behavior and prognosis after differentiated treatment in relation to type. Ann. Surg. 242, 64-73 (2005)

6. K. Borch, H. Renvall, G. Liedberg, Gastric endocrine cell hyperplasia and carcinoid tumors in pernicious anemia. Gastroenterology 88, 638-648 (1985)

7. G. Rindi, C. Azzoni, S. La Rosa, C. Klersy, D. Paolotti, S. Rappel, M. Stolte, C. Capella, C. Bordi, E. Solcia, ECL cell tumor and poorly differentiated endocrine carcinoma of the stomach: prognostic evaluation by pathological analysis. Gastroenterology 116, 532-542 (1999)

8. O. Hosokawa, Y. Kaizaki, M. Hattori, K. Douden, H. Hayashi, M. Morishita, K. Ohta, Long-term follow up of patients with multiple gastric carcinoids associated with type A gastritis. Gastric Cancer 8, 42-46 (2005)

9. D. Campana, F. Nori, R. Pezzilli, L. Piscitelli, D. Santini, E. Brocchi, R. Corinaldesi, P. Tomassetti, Gastric endocrine tumors type I: treatment with long-acting somatostatin analogs. Endocr. Relat. Cancer 15, 337-342 (2008)

10. E. Merola, A. Sbrozzi-Vanni, F. Panzuto, G. D’Ambra, E. Di Giulio, E. Pilozzi, G. Capurso, E. Lahner, C. Bordi, B. Annibale, 
G. Delle Fave, Type I gastric carcinoids: a prospective study on endoscopic management and recurrence rate. Neuroendocrinology 95, 207-213 (2012)

11. G. Delle Fave, D.J. Kwekkeboom, E. Van Cutsem, G. Rindi, B. Kos-Kudla, U. Knigge, H. Sasano, P. Tomassetti, R. Salazar, P. Ruszniewski, ENETS Consensus Guidelines for the management of patients with gastroduodenal neoplasms. Neuroendocrinology 95, 74-87 (2012)

12. S. Rappel, A. Altendorf-Hofmann, M. Stolte, Prognosis of gastric carcinoid tumours. Digestion 56, 455-462 (1995)

13. K. Hori, H. Fukui, J. Imura, T. Kojima, M. Fujita, H. Kawamata, T. Chiba, T. Fujimori, Benign gastric carcinoid tumor with hypergastrinemia followed up for 12 years. Gastric Cancer 3, 161-164 (2000)

14. D. Ravizza, G. Fiori, C. Trovato, N. Fazio, G. Bonomo, F. Luca, L. Bodei, G. Pelosi, D. Tamayo, C. Crosta, Long-term endoscopic and clinical follow-up of untreated type 1 gastric neuroendocrine tumours. Dig. Liver Dis. 39, 537-543 (2007)

15. S. Grozinsky-Glasberg, G. Kaltsas, C. Gur, E. Gal, D. Thomas, S. Fichman, K. Alexandraki, D. Barak, B. Glaser, I. Shimon, D.J. Gross, Long-acting somatostatin analogues are an effective treatment for type 1 gastric carcinoid tumours. Eur. J. Endocrinol. 159, 475-482 (2008)

16. S. Manfredi, M. Pagenault, A.S. de Lajarte-Thirouard, J.F. Bretagne, Type 1 and 2 gastric carcinoid tumors: long-term followup of the efficacy of treatment with a slow-release somatostatin analogue. Eur. J. Gastroenterol. Hepatol. 19, 1021-1025 (2007)

17. A. Uygun, A. Kadayifci, Z. Polat, K. Yilmaz, A. Gunal, H. Demir, S. Bagci, Long-term results of endoscopic resection for type I gastric neuroendocrine tumors. J. Surg. Oncol. 109, 71-74 (2014)

18. D. Thomas, A.V. Tsolakis, S. Grozinsky-Glasberg, M. Fraenkel, K. Alexandraki, S. Sougioultzis, D.J. Gross, G. Kaltsas, Longterm follow-up of a large series of patients with type 1 gastric carcinoid tumors: data from a multicenter study. Eur. J. Endocrinol. 168, 185-193 (2013)

19. Y. Sato, H. Imamura, Y. Kaizaki, W. Koizumi, K. Ishido, K. Kurahara, H. Suzuki, J. Fujisaki, K. Hirakawa, O. Hosokawa, M.
ItoM. Kaminishi, T. Furuta, T. Chiba, K. Haruma, Management and clinical outcomes of type I gastric carcinoid patients: retrospective, multicenter study in Japan. Dig. Endosc. 26(3), 377-384 (2013)

20. M.F. Dixon, R.M. Genta, J.H. Yardley, P. Correa, Classification and grading of gastritis. The updated Sydney System. International Workshop on the Histopathology of Gastritis, Houston 1994. Am. J. Surg. Pathol. 20, 1161-1181 (1996)

21. F.T. Bosman, F. Carneiro, R.H. Hruban, N.D. Theise, WHO classification of tumors of the digestive system, 4th edn. (IARC, Lyon, 2010)

22. G. Rindi, G. Kloppel, H. Alhman, M. Caplin, A. Couvelard, W.W. de Herder, B. Erikssson, A. Falchetti, M. Falconi, P. Komminoth, M. Korner, J.M. Lopes, A.M. McNicol, O. Nilsson, A. Perren, A. Scarpa, J.Y. Scoazec, B. Wiedenmann, TNM staging of foregut (neuro)endocrine tumors: a consensus proposal including a grading system. Virchows Arch. 449, 395-401 (2006)

23. V. Fykse, A.K. Sandvik, G. Qvigstad, S.E. Falkmer, U. Syversen, H.L. Waldum, Treatment of ECL cell carcinoids with octreotide LAR. Scand. J. Gastroenterol. 39, 621-628 (2004)

24. V. Fykse, A.K. Sandvik, H.L. Waldum, One-year follow-up study of patients with enterochromaffin-like cell carcinoids after treatment with octreotide long-acting release. Scand. J. Gastroenterol. 40, 1269-1274 (2005)

25. A. Rinke, H.H. Muller, C. Schade-Brittinger, K.J. Klose, P. Barth, M. Wied, C. Mayer, B. Aminossadati, U.F. Pape, M. Blaker, J. Harder, C. Arnold, T. Gress, R. Arnold, Placebo-controlled, double-blind, prospective, randomized study on the effect of octreotide LAR in the control of tumor growth in patients with metastatic neuroendocrine midgut tumors: a report from the PROMID Study Group. J. Clin. Oncol. 27, 4656-4663 (2009)

26. S. La Rosa, F. Inzani, A. Vanoli, C. Klersy, L. Dainese, G. Rindi, C. Capella, C. Bordi, E. Solcia, Histologic characterization and improved prognostic evaluation of 209 gastric neuroendocrine neoplasms. Hum. Pathol. 42, 1373-1384 (2011) 